\title{
The de Morton Mobility Index (DEMMI): An essential health index for an ageing world
}

\author{
Natalie A de Morton*1,2, Megan Davidson ${ }^{3}$ and Jennifer L Keating ${ }^{1}$
}

Address: ${ }^{1}$ Department of Physiotherapy, School of Primary Health Care, Faculty of Medicine, Nursing and Health Sciences, Monash University Peninsula Campus, PO Box 527, Frankston, Victoria, 3199, Australia, ${ }^{2}$ The Northern Clinical Research Center, Northern Health, 185 Cooper St, Epping, Victoria, 3076, Australia and ${ }^{3}$ School of Physiotherapy, Division of Allied Health, Faculty of Health Sciences, La Trobe University, Victoria, 3086, Australia

Email: Natalie A de Morton* - natalie.demorton@med.monash.edu.au; Megan Davidson - m.davidson@latrobe.edu.au; Jennifer L Keating - jenny.keating@med.monash.edu.au

* Corresponding author

Published: 19 August 2008

Health and Quality of Life Outcomes 2008, 6:63 doi:10.1 186/1477-7525-6-63

This article is available from: http://www.hqlo.com/content/6/l/63

This is an Open Access article distributed under the terms of the Creative Commons Attribution License (http://creativecommons.org/licenses/by/2.0), which permits unrestricted use, distribution, and reproduction in any medium, provided the original work is properly cited.

\begin{abstract}
Background: Existing instruments for measuring mobility are inadequate for accurately assessing older people across the broad spectrum of abilities. Like other indices that monitor critical aspects of health such as blood pressure tests, a mobility test for all older acute medical patients provides essential health data. We have developed and validated an instrument that captures essential information about the mobility status of older acute medical patients.

Methods: Items suitable for a new mobility instrument were generated from existing scales, patient interviews and focus groups with experts. $5 \mathrm{I}$ items were pilot tested on older acute medical inpatients. An interval-level unidimensional mobility measure was constructed using Rasch analysis. The final item set required minimal equipment and was quick and simple to administer. The de Morton Mobility Index (DEMMI) was validated on an independent sample of older acute medical inpatients and its clinimetric properties confirmed.

Results: The DEMMI is a 15 item unidimensional measure of mobility. Reliability $\left(M^{2} C_{90}\right.$ ), validity and the minimally clinically important difference (MCID) of the DEMMI were consistent across independent samples. The $\mathrm{MDC}_{90}$ and $\mathrm{MCID}$ were 9 and 10 points respectively (on the 100 point Rasch converted interval DEMMI scale).

Conclusion: The DEMMI provides clinicians and researchers with a valid interval-level method for accurately measuring and monitoring mobility levels of older acute medical patients. DEMMI validation studies are underway in other clinical settings and in the community. Given the ageing population and the importance of mobility for health and community participation, there has never been a greater need for this instrument.
\end{abstract}

\section{Background}

Contemporary beliefs are that physical decline is not the natural partner of aging and that people can remain physically able and independent for the duration of their lives.
This progressive position is reflected in encouragement of regular exercise and activity in older people $[1,2]$. However, by systematically reviewing existing instruments, we identified that a broadly applicable instrument that accu- 
rately measures and monitors mobility of older adults across the spectrum of health does not exist [3]. In this systematic review, the Elderly Mobility Scale (EMS) [4], Hierarchical Assessment of Balance and Mobility (HABAM) [5] and the Physical Performance Mobility Examination (PPME) [6] were identified as potentially suitable. However, clinimetric evaluation indicated significant limitations with each of these mobility instruments.

The HABAM, EMS and PPME were each designed for measuring the mobility of hospitalised older patients. Following clinimetric evaluation [3], the HABAM was identified to have the most desirable properties of these existing instruments. However, an important limitation of the HABAM is a ceiling effect $(25 \%$ of persons scoring the highest possible score) in an older acute medical population [5]. These findings support the proposal that a new mobility instrument is required for older acute medical patients.

Two common instruments for assessing mobility in the acute hospital environment are the Timed Up and Go test (TUG) [7] and the Barthel Index (BI)[8]. However, these instruments have inadequate scale width [9-13] to capture changes in physical health for people whose limitations are either severe or relatively modest. The TUG has a floor effect with approximately one quarter of patients unable to complete this test because they are too weak [10] and the BI has a ceiling effect with approximately one quarter of patients scoring within the error margin of the highest score [10].

Mobility is an important indicator of the health status of older people. According to the World Health Organisation's International Classification of Functioning (ICF) [14] 'mobility' is classified as one of nine domains of 'activity and participation' and is defined as "moving by changing body position or location or by transferring from one place to another, by carrying moving or manipulating objects, by walking, running or climbing, and by using various forms of transportation."

Without an accurate mobility instrument, healthcare providers cannot accurately monitor deterioration in mobility and appropriate strategies to maintain physical health may not be triggered. It has been argued that inadequate measures of physical ability, across the spectrum of abilities that exist in older people, presents the most pressing issue in exercise gerontology [15]. It has also been suggested that until such measures exist, our understanding of particular aspects of physical ageing will be limited [16].

Hospitalised people have a diverse range of acute clinical presentations and co-morbid conditions. The primary aim of this research was to develop a practical and high quality instrument with the scale width for measuring the mobility status of all hospitalised older medical patients. A fundamental aspect of instrument design was that data would be based on observation of performance rather than patient or proxy recall of mobility to avoid distortion associated with poor recall or cognitive deficits [17].

\section{Methods}

The four phases in instrument development were approved by the Ethics Committees at The Northern Hospital and/or Monash University.

\section{Phase I: Item generation and development}

Items were generated from existing mobility scales, 3 focus groups with academics and clinicians from relevant healthcare disciplines $(n=24)$ and patient interviews $(n=$ 12 ). Items were sought that assessed older people across the spectrum of mobility from bed bound to fully active and the search for relevant items continued to the point where additional information became redundant. Two independent assessors applied pre-determined criteria. To be included, it was necessary that the item

- was able to be easily administered i.e. can be performed at the patient's bedside

- was brief to conduct

- was administered based on observation of patient performance

- could be administered by professionals from different healthcare professions

- was appropriate to administer in an acute care hospital

- could be safely administered to patients who have an acute medical condition

- required minimal equipment

- provided measurable information about patient mobility

- provided objective information about patient mobility that would facilitate goal setting

for treatment

- administration could be clearly and unambiguously defined

- provided information that was not duplicated by another item 
Using consensus of experts, unambiguous and practical testing protocols were developed for 51 mobility items that remained after two independent assessors removed redundant items and applied inclusion criteria.

\section{Phase 2: Item testing \\ Participants}

Participants were recruited from general medical wards at The Northern Hospital, Victoria, Australia. Consecutive participants were screened by a recruiting officer and were eligible to participate if 65 years or older and were assessed within 48 hours of admission. Patients were excluded if they had a planned hospital stay of less than 48 hours, severe dysphasia, documented contra-indications to mobilization, were isolated for infection, or if death was imminent. All eligible participants were invited to participate. Consent was obtained within 48 hours of hospital admission. For patients deemed incompetent to consent, this was obtained from the 'person responsible' or next-of-kin. Interpreters were employed when required.

\section{Testing procedure}

Participants were assessed at the bedside every 48 hours during hospitalisation or on the Monday following a weekend. Baseline measurements included age, sex, place of residence prior to admission, primary language, gait aid use prior to hospitalisation, Mini Mental State Examination (MMSE) [18], Charlson Comorbidity Index [19], APACHE11 Severity of Illness Scale [20], the Barthel Index (BI) $[8,21]$, Hierarchical Assessment of Balance and Mobility (HABAM) [5] and the new mobility items. The BI and HABAM were selected for a head-to-head comparison with the new mobility instrument. The BI is widely used as a self report measure of independence in activities of daily living in the acute hospital setting [11] and, prior to this study, the HABAM was identified as having the most desirable properties of existing mobility instruments [3]. Each of these outcome measures are described in further detail below.

At each assessment a researcher administered the BI and the MMSE. As close as possible to this assessment, the patient was assessed on the mobility items by the principal researcher, who was blind to BI scores. The HABAM items were a subset of these mobility items.

Mobility items were administered in the order of bed, chair, balance and walking activities to maximise patient safety, confidence and ease of testing. Familiarisation trials were not provided to minimise fatigue and time required to administer the test. At each test the therapist and patient independently rated the patient's current mobility compared with admission mobility on a 5 point scale (much worse, bit worse, same, bit better, much better). This provided a reference standard for important change in mobility.

\section{Outcome measures}

The APACHE 11 is a severity of illness scale with a score range from 0 to 71 , where higher scores represent increasing severity of illness during the first 24 hours of hospital admission. The Charlson Index classifies comorbid conditions according to risk of mortality. One year mortality rates in a medical population have been reported to be $12 \%, 26 \%, 52 \%$ and $85 \%$ for Charlson scores of $0,1-2$, 3-4 and greater than 5 respectively [19].

The modified $\mathrm{BI}$ is an ordinal scale that provides a total score between 0 and 100 where higher scores indicate greater independence in activities of daily living [21]. The HABAM is an interval level mobility instrument that provides a score between 0 and 26 [5] where higher scores indicate increasing levels of independent mobility and was designed for application in an older acute medical population. The MMSE is reported to be a valid and reliable measure of patient cognition [18]. It provides a score between 0 and 30 points where increasing scores indicate better cognitive ability.

\section{Item reduction}

The complete set of 51 mobility items were pilot tested for two weeks to remove items with practical limitations, a process that included patient and assessor interview about the mobility tests. The remaining 42 items were then tested on a large sample by the principal researcher. After completion, items with practical limitations were removed and Rasch analysis conducted.

\section{Rasch analysis}

Data analyses were performed using SPSS version 12.0 [22] and RUMM2020 [23]. The Rasch partial credit model was employed to identify misfitting and redundant items and to identify a hierarchy of mobility items ranked from easiest to hardest. Participants were divided into 3 class intervals (ie, 3 groups of patients at different levels of mobility). Item misfit was considered if the chi-square or F statistic probability value was less than the Bonferroniadjusted a value for multiple testing or the fit residuals were greater than \pm 2 .

Item residuals from Rasch analysis were also examined as a finding of no association between residuals for individual items has been argued as evidence of local item independence [24]. High positive correlation between residuals provides evidence of local item dependence and high negative correlations is thought to indicate multidimensionality.

Differential item functioning (DIF) analysis [25] was planned for age, gender, time of assessment, cognitive status (MMSE) and whether an interpreter was required. DIF was considered significant if the chi-square probability value was lower than the Bonferroni-adjusted $p$ value. $A$ 
priori, these factors were considered potential confounders to item functioning.

Item response thresholds were also studied to investigate the existence of disordered thresholds, that is, response patterns on the rating scale that are not in the expected order. The person separation index (PSI) was reported to provide an indication of the internal consistency (reliability) of the scale by examining the ability of the instrument to discriminate among respondents.

Sample size for Rasch analysis was based on recommendations by Linacre et al [26]. These authors recommend a sample size of $64-144$ to provide $95 \%$ confidence + - 0.5 logits. Baseline and 48 hour assessments during a 3-4 month period were expected to provide more than 200 assessments. In the absence of DIF by time, all available assessments would be included for Rasch analysis as recommended by Wright [27] and Chang and Chang [28].

\section{Phase 3: Interval scoring system and clinimetric evaluation (development sample)}

Based on Rasch analysis, an interval scoring system (0100) was developed to facilitate clinical application and clinimetric evaluation of the reduced item set.

\section{Reliability study}

An inter-rater reliability study was conducted on a subset of patients who reported no fatigue from the first assessment. After the first assessment and a 10 minute rest, the mobility assessment was repeated by a physiotherapist blind to the outcomes of the first test. Test order of assessing physiotherapists was randomised. Power calculations were performed based on recommendations by Walter et al [29]. The Minimal Detectable Change at $90 \%$ confidence $\left(\mathrm{MDC}_{90}\right)$ and accompanying $95 \%$ confidence intervals were estimated [30].

\section{Validity}

Correlation coefficients and associated 95\% confidence intervals were calculated to investigate the convergent validity of DEMMI scores with the BI (a measure of a related construct) and HABAM (a measure of the same construct), and discriminant validity with the MMSE, Charlson Index and APACHE 11 (measures of different constructs). To investigate known-groups validity, an independent $t$ test was performed on DEMMI scores of patients discharged to home compared to inpatient rehabilitation.

\section{Minimum clinically important difference}

The MCID was calculated for DEMMI, HABAM and BI as the mean change score for patients who rated themselves 'much better' at discharge (criterion based method). The MCID was also calculated using distribution based method recommended by Norman et al[31].

\section{Responsiveness to change}

The Effect Size Index (distribution method)(ESI) and Guyatt's Responsiveness Index (criterion method)(GRI), were selected a priori to calculate measurement responsiveness of the DEMMI, HABAM and BI. Inferential 95\% confidence bands were calculated to enable statistical comparison of responsiveness estimates as recommended by Tryon [32].

\section{Time to administer}

The time required to administer the DEMMI was rounded to the nearest 30 seconds and was recorded using a stop watch.

\section{Phase 4: Final DEMMI refinement and validation in an independent sample}

Prior to testing in an independent sample, the DEMMI was administered by clinicians from several health care disciplines. Clinician responses to a set of structured, oneon-one interview questions were used to refine the instrument format, items and testing protocol.

The refined instrument was then tested on an independent sample of older acute medical patients and evaluated, as per phases 2 and 3. An independent physiotherapist (not involved in the instrument development) conducted the mobility assessments.

\section{Results}

The stages of instrument development in this study are summarised in Figure 1.

\section{Phase I: Item generation and development}

Ninety seven mobility items were generated from focus groups and 75 items from existing mobility instruments. One additional item was generated from patient interviews. After removal based on item duplication, redundancy and application of inclusion criteria, 51 items remained for pilot testing (Table 1 ).

\section{Phase 2: Item testing}

Pilot testing 5 I mobility items

Pilot testing on 15 consecutive older general medical patients identified 9 items for removal based on practical limitations (Table 1).

\section{Testing of 42 remaining mobility items}

Figure 2 shows that of the 388 new hospital admissions screened for inclusion, 219 were eligible, 104 were recruited and 89 performed at least one mobility assessment. Three patients were readmitted during the study period and were included twice as new hospital admissions. Table 2 shows the admission characteristics for the 86 patients included in this study. There were no adverse events as a result of the mobility assessments. A further 8 items were removed due to practical limitations that were 


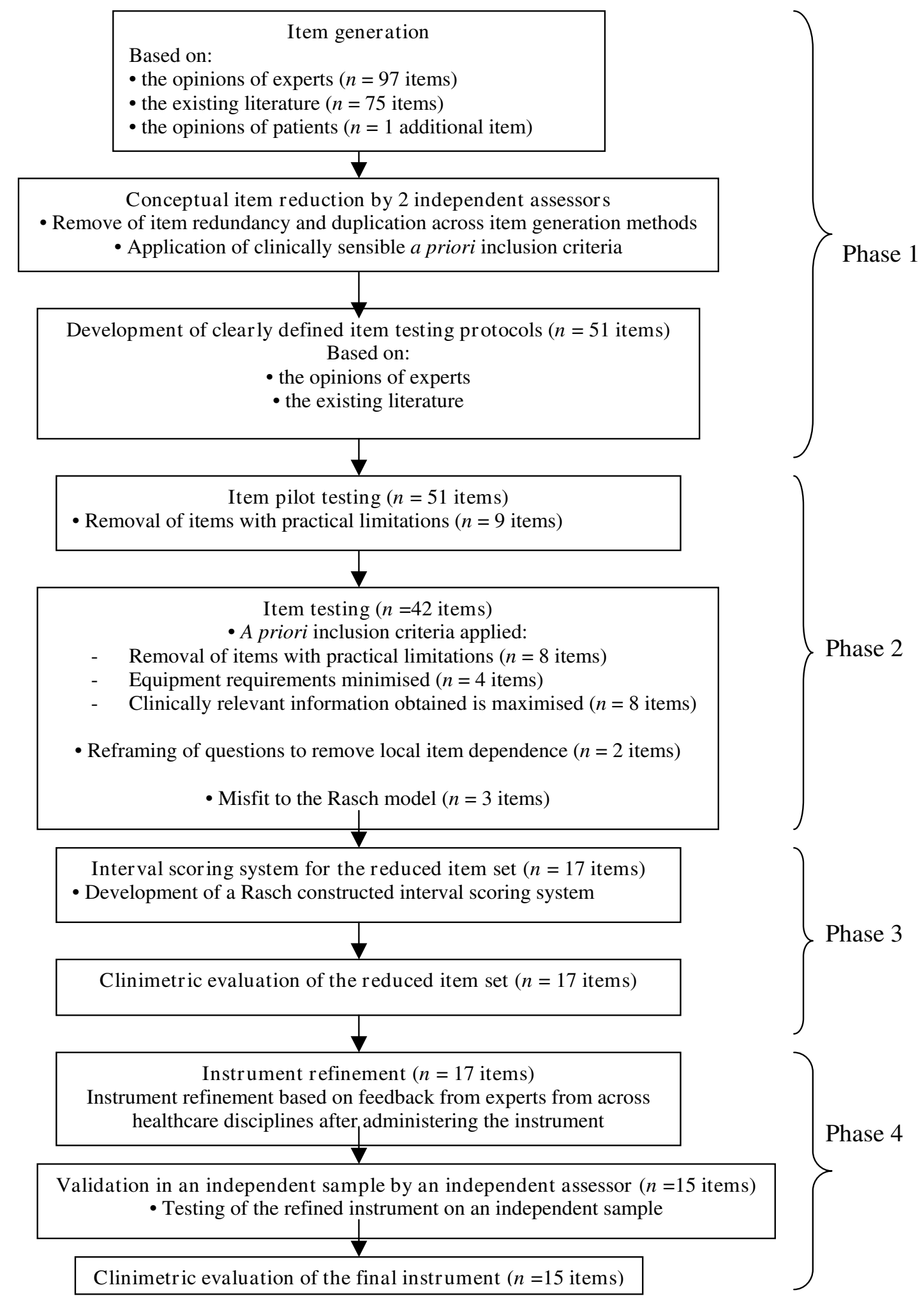

Figure I

Stages of unidimensional instrument development. 
Table I: Reasons for item exclusion at each stage of instrument development

\begin{tabular}{ll}
\hline Excluded item & Reason for exclusion
\end{tabular}

\section{Pilot testing of 5 I mobility items: 9 items excluded due to practical limitations}

Number of times in/out of bed in $10 \mathrm{sec}$

Sit to stand 3 times in 10 seconds

Sitting balance and turning head

Reach sideways to pick up pen from floor (sitting)

Reach sideways to pick up pen from floor (standing)

Walk 6 meters in 10 seconds

Step test

Step

Step over box
Removed to maximise patient safety. Difficult to test for patients who have drips, drains, indwelling catheters etc. A similar item, 'lying to sitting independently within 10 seconds' was deemed to be safer and provided similar clinical information.

To reduce the burden of testing by minimising redundancy of sit to stand items. 'Independent sit to stand in 3 seconds' was retained due to shorter administration time.

Many patients had significantly limited cervical range of movement and therefore this test was difficult to standardise across patients.

Several patients reported feeling dizzy performing this task after first attempting to reach forward to pick up pen from floor. Reaching forwards to pick up a pen was considered to be the more functional item and was therefore retained.

As above

Requires a standardised walking test environment which could not be relied upon.

Requires a standardised step. Removed due to equipment requirements. Requires a standardised step. Removed due to equipment requirements. Requires a standardised step. Removed due to equipment requirements.

\section{Testing of $\mathbf{4 2}$ mobility items: 8 items excluded due to practical limitations}

\section{Skipping}

Sit to stand using the chair seat (not using the arms of the chair)

Immediate standing balance

Semi tandem stance

Reach in sitting

360 degree turn

Sit to lie

Hop
This is a complex movement that required practice to perform in a standardised way.

For wider patients there was not enough space to push up from the seat. Cognitively impaired patients found this task difficult to understand when the arms of the chair were accessible.

Required significant explanation, particularly for cognitively impaired patients.

Required significant explanation and/or demonstration for patients to understand task.

Dizziness prevented some patients from successfully completing this item.

This item was difficult to perform with patients who had lines, drips, drains etc.

Asking the patient to return to bed to assess this item interrupted the flow of testing.

This is a dynamic single leg activity and was removed to maximise patient safety.

Reframing walking items to remove potential for local item dependence (assumption of Rasch analysis)

Four walking items: $5 \mathrm{~m}, 10 \mathrm{~m}, 20 \mathrm{~m}$ and $50 \mathrm{~m}$

(response options were levels of assistance for each distance)
4 walking items replaced with 2 items:

I. walks +/- gait aid (with distance response options)

2. walking assistance (with levels of assistance for response options)

Rasch analysis of 32 mobility items: 4 items removed

Transferring from bed to chair

Carrying a glass of water while walking

Timed bed transfer

Timed chair transfer
Required equipment and had similar threshold locations to other items Required equipment and had similar threshold locations to other items Required equipment and had similar threshold locations to other items Required equipment and had similar threshold locations to other items

Removal of items that provided similar clinical information (and to avoid local item dependence): 8 items removed 
Table I: Reasons for item exclusion at each stage of instrument development (Continued)

\begin{tabular}{|c|c|}
\hline & $\begin{array}{l}\text { 'Sitting unsupported' is a simpler test and maximises scale width as it has } \\
\text { the lowest logit item score (easiest item). }\end{array}$ \\
\hline$\times 5$ sit to stand without arms & $\begin{array}{l}\text { Similar items: } \times 1 \text { sit to stand without arms and } \times 5 \text { sit to stand without } \\
\text { arms. } \\
\text { ' } x \mid \text { sit to stand without arms' is a simpler and quicker test. }\end{array}$ \\
\hline Standing arm raise Standing with eyes closed & $\begin{array}{l}\text { Similar items: Standing unsupported, standing arm raise and standing } \\
\text { with eyes closed. } \\
\text { 'Standing unsupported' is the simplest test and is an important } \\
\text { component of independent mobility. }\end{array}$ \\
\hline Standing with feet together eyes closed & $\begin{array}{l}\text { Similar items: Standing with feet together and standing with feet } \\
\text { together eyes closed } \\
\text { 'Standing with feet together' is a simpler test. }\end{array}$ \\
\hline Tandem standing Tandem walking & $\begin{array}{l}\text { Similar items: Tandem standing, tandem standing with eyes closed and } \\
\text { tandem walking }\end{array}$ \\
\hline & $\begin{array}{l}\text { 'Tandem standing with eyes closed' had the second highest item logit } \\
\text { location (second most difficult item) and was therefore retained to } \\
\text { maximise scale width. }\end{array}$ \\
\hline Stand on one leg & $\begin{array}{l}\text { Similar items: Stand on one leg and stand on one leg eyes closed } \\
\text { 'Stand on one leg with eyes closed' had the highest item logit location } \\
\text { (most difficult item) and was therefore retained to maximise scale width. }\end{array}$ \\
\hline
\end{tabular}

Rasch analysis of $\mathbf{2 0}$ mobility items: $\mathbf{3}$ items removed

\begin{tabular}{ll}
\hline Toe walk & $\begin{array}{l}\text { Similar threshold locations to other items and statistically significant } \\
\text { misfit }\end{array}$ \\
Heel walk & $\begin{array}{l}\text { Similar threshold locations to other items and statistically significant } \\
\text { misfit } \\
\text { Similar threshold locations to other items and statistically significant } \\
\text { misfit }\end{array}$ \\
\hline
\end{tabular}

identified following further testing and the 4 walking items were rescored to 2 items to limit local item dependence (an assumption of Rasch analysis)(Table 1).

Rasch analysis of 32 mobility items

Following item testing and Rasch analysis, 32 items were reduced to 17 (Table 1 ). DIF by time was not identified for the 17 items and therefore Rasch analysis was performed on data from hospital admission and subsequent 48 hour assessments. Rescoring three items (lie to sit, sit to stand and walking distance) produced ordered thresholds for all items.

Data for the 17 mobility items fitted the Rasch model (item-trait $\chi^{2}=41.17, \mathrm{df}=34, \mathrm{p}=0.19$ ). The $\mathrm{t}$ test procedure $[24,33]$ identified that the percentage of individual $t$ tests outside the acceptable range was only $4.23 \%$. (95\% CI $1.0 \%$ to $7.0 \%$ ). This provides further evidence of the unidimensionality of the 17 mobility items.

Examination of the residual correlation matrix indicated negative correlations of greater than 0.3 between sit unsupported and bridge $(\mathrm{r}=-0.55)$, standing on toes and stand on one leg eyes closed $(\mathrm{r}=-0.58)$ and tandem standing eyes closed and walking distance $(\mathrm{r}=0.35)$. However, these findings were not supported by high fit residuals for any of these items. A positive correlation of greater than 0.30 was only identified between the roll and lie to sit $(\mathrm{r}=+0.37)$ items.
Although this result indicates the possibility of some response dependency between these mobility tasks, both items were retained as each provides important clinical information regarding patient mobility and care needs during acute hospitalisation. In addition, examination of the admission only dataset indicated a lower correlation of +0.21 .

Person separation was 0.92 , indicating the test could discriminate 5.8 strata of ability.

Phase 3: Interval scoring system and clinimetric evaluation Raw scores for the reduced item set were converted to a $0-$ 100 interval scale. The clinimetric properties for the 17 item DEMMI are reported in Table 3.

\section{Reliability}

Correlation between independent assessor DEMMI interval scores was high (Pearson's $r=0.94,95 \%$ CI 0.86 to $0.98)$. The mean scores for assessors 1 and 2 were 57.19 ( $\mathrm{sd}=17.07)$ and $55.05(\mathrm{sd}=13.77)$ points respectively. A paired t test indicated no systematic differences between assessors $(\mathrm{p}=0.14)$. Using a pooled standard deviation of 15.51, the standard error of measurement (SEM) was 4.10 and the inter-rater reliability $\mathrm{MDC}_{90}$ was 9.51 points (95\% CI 5.04 to 13.32 ) on the 100 point DEMMI interval scale. This indicates that a patient needs to improve or deteriorate by 10 points or more for a clinician to be $90 \%$ 
Table 2: Patient baseline demographics for the instrument development and validation

\begin{tabular}{|c|c|c|}
\hline Patient Baseline demographics & Development study $n=86$ & Validation study $n=106$ \\
\hline Mean Age years (sd) & $79.2(7.1)$ & $81.2(7.3)$ \\
\hline Gender (\% female) & $53 \%$ & $47.3 \%$ \\
\hline \multicolumn{3}{|l|}{ Place of prior residence } \\
\hline Home alone & $24(27.9 \%)$ & 31 (29.3\%) \\
\hline Home accompanied & $52(60.5 \%)$ & $65(61.3 \%)$ \\
\hline Hostel/SRS & $6(7 \%)$ & $8(7.6 \%)$ \\
\hline Nursing Home & $4(4.7 \%)$ & $2(1.9 \%)$ \\
\hline \multicolumn{3}{|l|}{ Primary Language } \\
\hline English & $59(68.6 \%)$ & 75 (69.8\%) \\
\hline Italian & $17(19.8 \%)$ & $14(13.2 \%)$ \\
\hline Macedonian & $3(3.5 \%)$ & I (0.9\%) \\
\hline Other & $7(8.1 \%)$ & $17(16.1 \%)$ \\
\hline \multicolumn{3}{|l|}{ Gait aid prior to hospital admission } \\
\hline None & $32(37.2 \%)$ & 50 (44.6\%) \\
\hline Walking stick & $26(30.2 \%)$ & $22(19.6 \%)$ \\
\hline Frame & $25(29.1 \%)$ & 37 (33\%) \\
\hline Other & $3(3.5 \%)$ & $3(2.7 \%)$ \\
\hline \multicolumn{3}{|l|}{ Primary Diagnosis } \\
\hline Circulatory & $20(23.3 \%)$ & $21(19.8 \%)$ \\
\hline Respiratory & $13(15.1 \%)$ & 37 (34.9\%) \\
\hline Endocrine & $9(10.5 \%)$ & $6(5.7 \%)$ \\
\hline Digestive & $4(4.7 \%)$ & $7(6.6 \%)$ \\
\hline Genitourinary & $4(4.7 \%)$ & $6(5.7 \%)$ \\
\hline Musculoskeletal & $4(4.7 \%)$ & $3(2.8 \%)$ \\
\hline Other & $32(37.2 \%)$ & $26(24.5 \%)$ \\
\hline Mean Charlson Index (sd) & $1.83(1.54), n=84$ & $1.94(1.57), n=105$ \\
\hline Mean APACHE II (sd) & $11.89(3.10), n=83$ & $12.60(3.77), n=105$ \\
\hline Mean MMSE (sd) & $21.73(7.57)$, range $0-30 n=85$ & $22.77(6.30)$, range $I-30, n=103$ \\
\hline Mean Barthel Index (sd) & 81.29 (22.72), range $20-100$ & $82.47(18.80)$, range $15-100, n=105$ \\
\hline Mean HABAM (sd) & I8.06 (6.78), range $0-26$ & $16.83(6.77)$, range $0-26$ \\
\hline
\end{tabular}

confident that a true change in patient condition has occurred. A paired t test indicated no systematic difference between the first and second assessment scores $(p=0.77)$.

\section{Validity}

DEMMI scores had a significant and high correlation with HABAM and BI scores. This provides evidence of convergent validity for the DEMMI.

Discriminant validity for the DEMMI was evidenced by a low correlation with measures of other constructs (MMSE, APACHE 11 severity of illness and Charlson co-morbidity index scores).

An independent $t$ test showed that patients who were discharged to inpatient rehabilitation had significantly lower DEMMI scores at acute hospital discharge than those discharged to home. Patients discharged to inpatient rehabilitation had a mean DEMMI score of 39.55 ( $\mathrm{sd}=9.41,95 \%$ CI 33.72 to 45.38 ) and patients discharged to home had a mean DEMMI score of 59.61 ( $\mathrm{sd}=13.22,95 \%$ CI 56.30 to 62.93 ). This provides evidence of known groups validity for the DEMMI.

\section{Responsiveness}

There was no significant difference identified between the responsiveness of DEMMI and HABAM measurements or DEMMI and BI measurements using the ESI or GRI based on patient or therapist report of change.

\section{Minimally clinically important difference}

By calculating the average change in DEMMI score for patients who reported to be 'much better' in their mobility between hospital admission and discharge, the MCID for the DEMMI was identified to be 8 points, that is, a change of 8 points or more is likely to represent a patient perceived important change in mobility. Using Norman et al.'s [31] distribution based method, the MCID was also calculated to be 8 points for the DEMMI.

\section{Phase 4: Final DEMMI refinement and validation in an independent sample \\ Item refinement}

Feedback from 15 clinicians was obtained following their administration of the DEMMI. Minor changes were made to the sit unsupported item and testing protocol and the final format of the DEMMI. 


\section{8 new hospital admission patients screened}

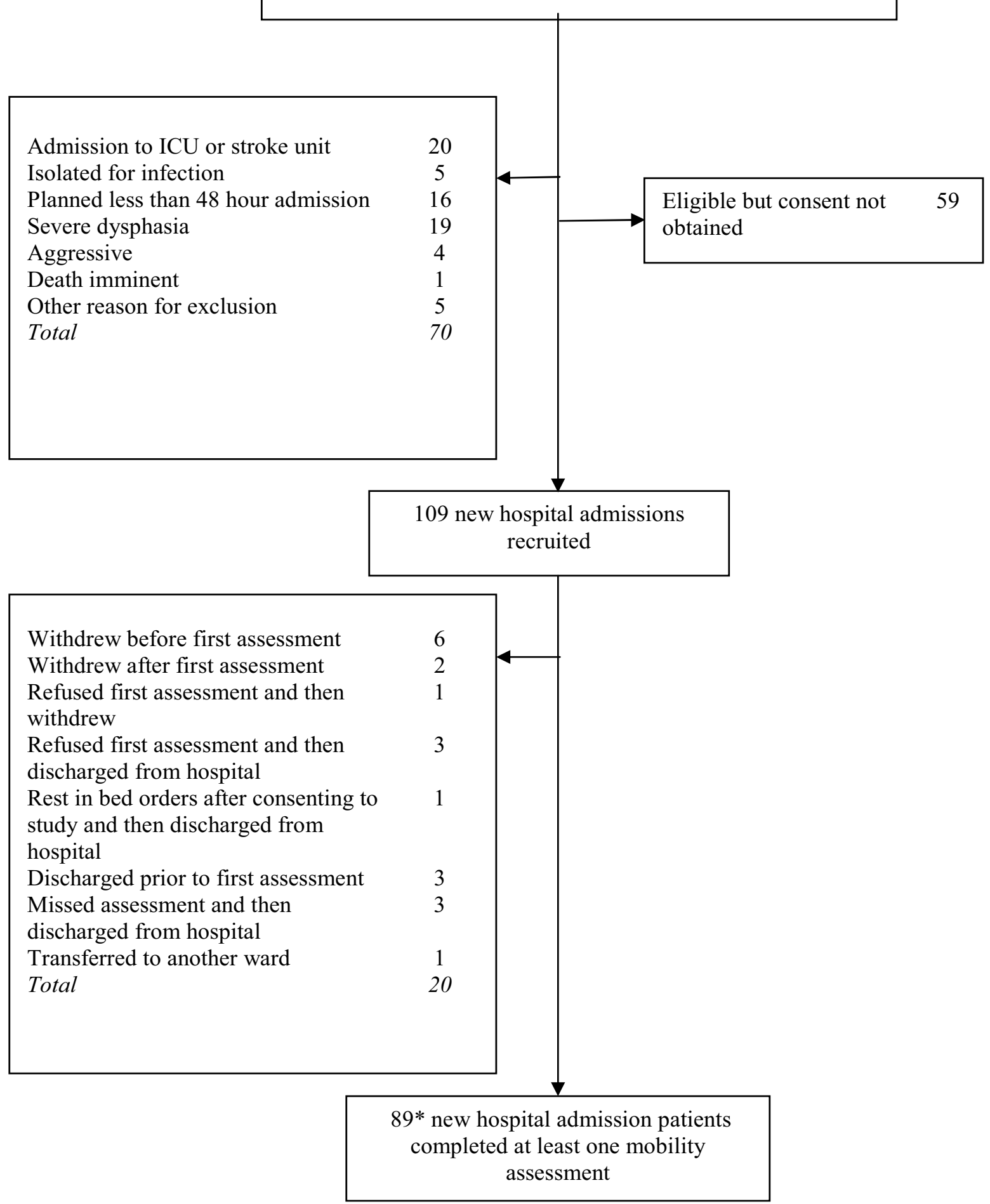

Figure 2

Development sample: flow of participants through the study. *3 patients were readmitted during the study period and were tested twice as 'new admissions.' 
Table 3: Clinimetric properties of the DEMMI

\begin{tabular}{|c|c|c|}
\hline Clinimetric property & Development study I 7 items & Validation study I 5 items \\
\hline \multicolumn{3}{|l|}{ Reliability, $\mathrm{MDC}_{90}(95 \% \mathrm{Cl})$} \\
\hline Inter rater & 9.5 (5.0 to 13.3$), n=21$ & 8.90 (6.3 to 12.7$), n=35$ \\
\hline \multicolumn{3}{|l|}{ MCID (95\%CI) } \\
\hline Criterion based method & 7.8 (5.3 to 10.2$)$ & 9.43 (5.9 to 12.9) \\
\hline Distribution based method & 8.0 & 10.5 \\
\hline \multicolumn{3}{|l|}{ Construct Validity $(r, 95 \% \mathrm{Cl})$} \\
\hline \multicolumn{3}{|l|}{ Convergent } \\
\hline HABAM & $0.92(0.88$ to 0.95$), p=0.00$ & 0.91 ( 0.87 to 0.94$), p=0.00$ \\
\hline Barthel Index & $0.76(0.65$ to 0.84$), p=0.00$ & 0.68 (0.56 to 0.77$), p=0.00$ \\
\hline \multicolumn{3}{|l|}{ Discriminant } \\
\hline MMSE & $0.36(0.16$ to 0.53$), p=0.00$ & $0.24(0.05$ to $0.4 I), p=0.02$ \\
\hline APACHE II & $-0.11(-0.32$ to 0.11$), p=0.18$ & $0.07(-0.12$ to 0.26$), p=0.49$ \\
\hline Charlson & $-0.19(-0.39$ to 0.03$), p=0.11$ & $-0.04(-0.23$ to 0.15$), p=0.68$ \\
\hline \multicolumn{3}{|l|}{ Known Groups (DEMMI, 95\%Cl) } \\
\hline discharge to rehabilitation & 37.54 (33.99 to 45.10$), n=11$ & $50.75(42.39$ to 59.11$) n=8$ \\
\hline discharge to home & $\begin{array}{l}59.61 \text { ( } 56.32 \text { to } 62.90), n=62 \\
\text { Independent } t \text { test: } p=0.00\end{array}$ & $\begin{array}{l}62.14(57.80 \text { to } 66.49) n=70 \\
\text { Independent } t \text { test: } p=0.03\end{array}$ \\
\hline \multicolumn{3}{|l|}{ Responsiveness to change ${ }^{\#}$} \\
\hline \multicolumn{3}{|l|}{ Effect Size Index } \\
\hline DEMMI & 0.37 (0.28 to 0.46$)$ & $0.39(0.28 \text { to } 0.50)^{*}$ \\
\hline HABAM & 0.31 ( 0.20 to 0.43$)$ & $0.35(0.23$ to 0.47$)$ \\
\hline Barthel Index & $0.30(0.17$ to 0.44$)$ & $0.13(0.01 \text { to } 0.25)^{*}$ \\
\hline \multicolumn{3}{|l|}{ GRI (patient)\# } \\
\hline DEMMI & $1.23(0.90$ to 1.56$)$ & $0.92(0.66 \text { to } 1.17)^{*}$ \\
\hline HABAM & $1.00(0.46$ to 1.55$)$ & $0.72(0.49$ to 0.94$)$ \\
\hline Barthel Index & $0.48(0.01$ to 0.95$)$ & $0.43(0.21 \text { to } 0.65)^{*}$ \\
\hline \multicolumn{3}{|l|}{ GRI (therapist)\# } \\
\hline DEMMI & 2.06 (I.60 to $2.5 \mathrm{I})$ & 1.73 (I.37 to 2.09$)^{*}$ \\
\hline HABAM & $2.62(1.70$ to 3.54$)$ & 1.17 (0.86 to I.48) \\
\hline Barthel Index & $1.58(0.56$ to 2.60$)$ & $0.65(0.37 \text { to } 0.93)^{*}$ \\
\hline Floor effect & $0 \%$ & $<1 \%$ \\
\hline Ceiling effect & $<1 \%$ & $3.8 \%$ \\
\hline Time to administer, mean (sd) & I 3 mins 42 seconds ( 4.99 mins) for 42 mobility items & $\begin{array}{c}8 \text { mins } 47 \text { seconds } \begin{array}{c}(3.89 \text { minutes) for } 17 \text { mobility } \\
\text { items }\end{array}\end{array}$ \\
\hline
\end{tabular}

GRI = Guyatt's Responsiveness Index, \# Tryon's inferential confidence intervals

* significant difference: evidenced by non overlapping inferential confidence intervals

Validation in an independent sample

Figure 3 shows that of 344 new hospital admissions screened, 216 were eligible, 132 were recruited and 112 performed at least one mobility assessment. Six patients were readmitted during the study period and were included twice as new hospital admissions. Another six patients did not complete a hospital admission assessment. Table 2 shows the admission characteristics for the 106 patients included in this study. A total of 312 mobility assessments were performed using the 17 mobility items. Patients in the validation study did not differ from the instrument development sample on any baseline characteristic.

Prior to conducting Rasch analysis the jog item was removed. This item required clinical experience of medical conditions to determine whether testing should proceed. No participant was able to successfully complete the standing on one leg with eyes closed item in the validation study. Rasch analysis was therefore performed for the remaining 15 items.

In the validation study, the pooled dataset showed misfit to the Rasch model due to large sample size as there was no evidence of DIF by time or multidimensionality. Using the t test procedure [24,33], multidimensionality was not identified. Four items (reaching for pen, backward walking, standing on toes and sit to stand no arms) had a positive correlation of 0.3 or greater and three items (walking distance, roll and lie-sit) had a negative correlation of 0.3 or greater with the first residual component. The t test procedure indicated the percentage of individual t tests outside the acceptable range was $4.88 \%$ (95\% CI $-2.0 \%$ to $7.0 \%)$. This provides further evidence of the unidimensionality of the 15 DEMMI items and therefore does not explain the misfit of the data to the Rasch model. No evidence of local item 


\section{4 new hospital admission patients screened}

Admission to ICU or stroke unit

Isolated for infection

Planned less than 48 hour admission

Severe dysphasia

Aggressive

Death imminent

Interpreter not available

Other

Total
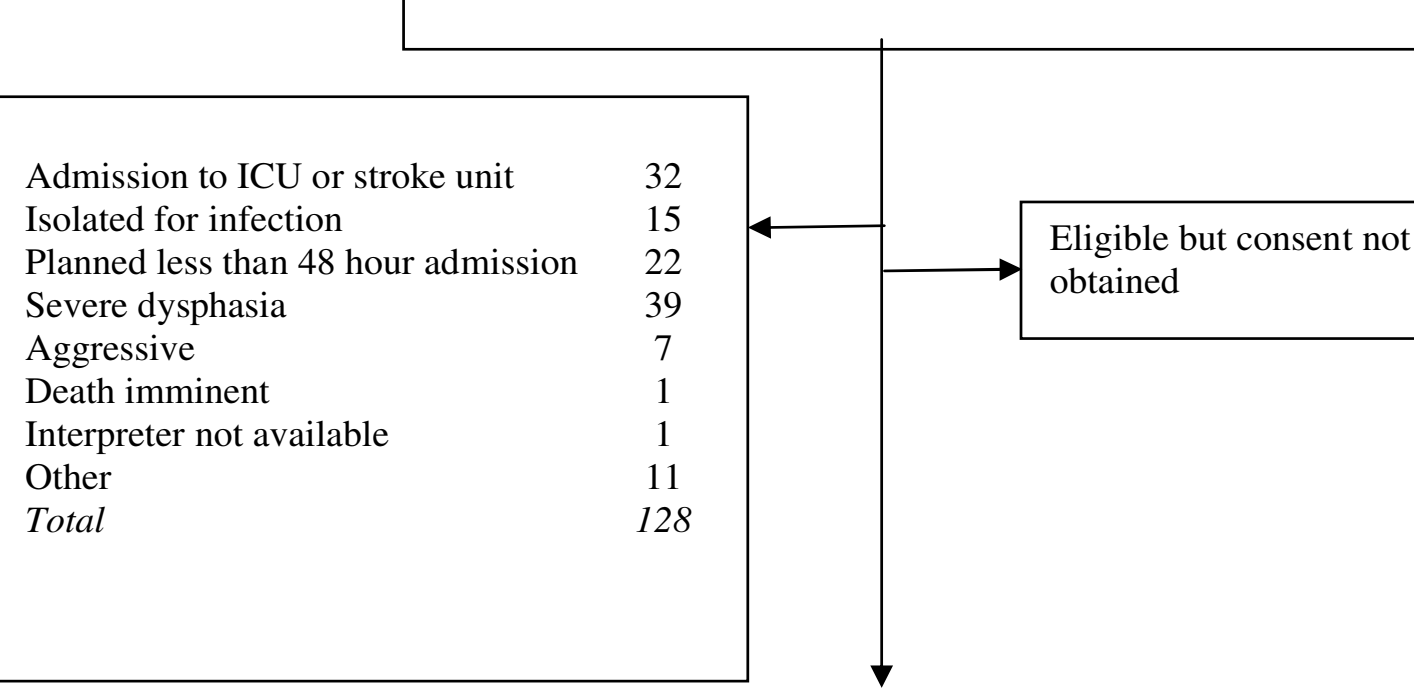

15

22

39

7

1

1

11

128

132 new hospital

admissions recruited

Withdrew before first assessment

Withdrew after first assessment

Refused first assessment and then

withdrew

Refused first assessment and then

discharged from hospital

Discharged prior to first assessment

Missed first assessment and then

discharged from hospital

First assessment unable to be

conducted and then discharged from

hospital

Transferred to another unit

Total

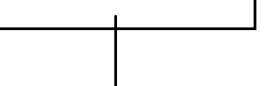

Eligible but consent not

obtained

.

$112^{* \#}$ new hospital admission patients completed at least one mobility assessment

\section{Figure 3}

Validation sample: flow of participants through the study. * 6 patients were readmitted during the study period and were tested twice as 'new admissions.' \# 106 'new admission' patients ( 100 patients) completed a hospital admission assessment (6 patients did not perform an admission assessment) 
dependency was identified as there was an absence of correlations in the residuals above a magnitude of 0.3 .

Data fitted the model at each assessment time point; baseline $\left(\chi^{2}=24.60, \mathrm{df}=30, \mathrm{p}=0.74\right)$, first 48 hour assessment $\left(\chi^{2}=36.37, \mathrm{df}=30, \mathrm{p}=0.20\right)$ and subsequent 48 hour assessments $\left(\chi^{2}=36.26, \mathrm{df}=28, \mathrm{p}=0.14\right)$. Given the similar findings across samples, analysis of hospital admission data is reported.

There were 106 hospital admission mobility assessments performed. The mobility items were well targeted for older acute medical patients. Figure 4 shows the average item difficulty and the person ability locations on the logit scale. There were only a few persons with a lower ability than the easiest item (to the left of the scale), or with a higher ability than the hardest item (to the right of the scale). None of the items showed misfit to the model and $\chi^{2}$ and $\mathrm{F}$ statistic Bonferroni adjusted probability values for the 15 items were non significant. Person separation was 0.88 , indicating the test could reliably identify 3.7 strata of ability.

Three items showed significant DIF by age but appear to be statistical artifacts due to a small number of participants in one of the three class intervals. Two items (lie to sit and walking independence) showed mild disordering of one threshold. However, inspection of item thresholds in the pooled dataset showed these items to be ordered and they were not rescored.

Figure 5 shows the item hierarchy for the 15 DEMMI items was consistent across independent samples. The final DEMMI is shown in Additional file 1 and its clinimetric properties reported in Table 3. The measurement properties of the DEMMI (Rasch, reliability, validity and MCID) were consistent with estimates obtained from the instrument development sample.

\section{Discussion}

The DEMMI provides clinicians and researchers with an advanced, practical and reliable instrument for measuring mobility in hospitalised older acute medical patients. The DEMMI is a unidimensional instrument that measures mobility across the spectrum from bed bound to independent mobility. It is safe, quick and easy to administer, has minimal equipment requirements, can be administered at a patient's bedside and provides interval data.

The DEMMI overcomes ceiling effects identified in the BI and HABAM and the floor effect identified in the Timed Up and Go in an older acute medical patient population. The DEMMI items cover the broad spectrum of mobility levels that exist for older acute general medical patients as neither ceiling nor floor effect were identified. Therefore this instrument has the width required to measure

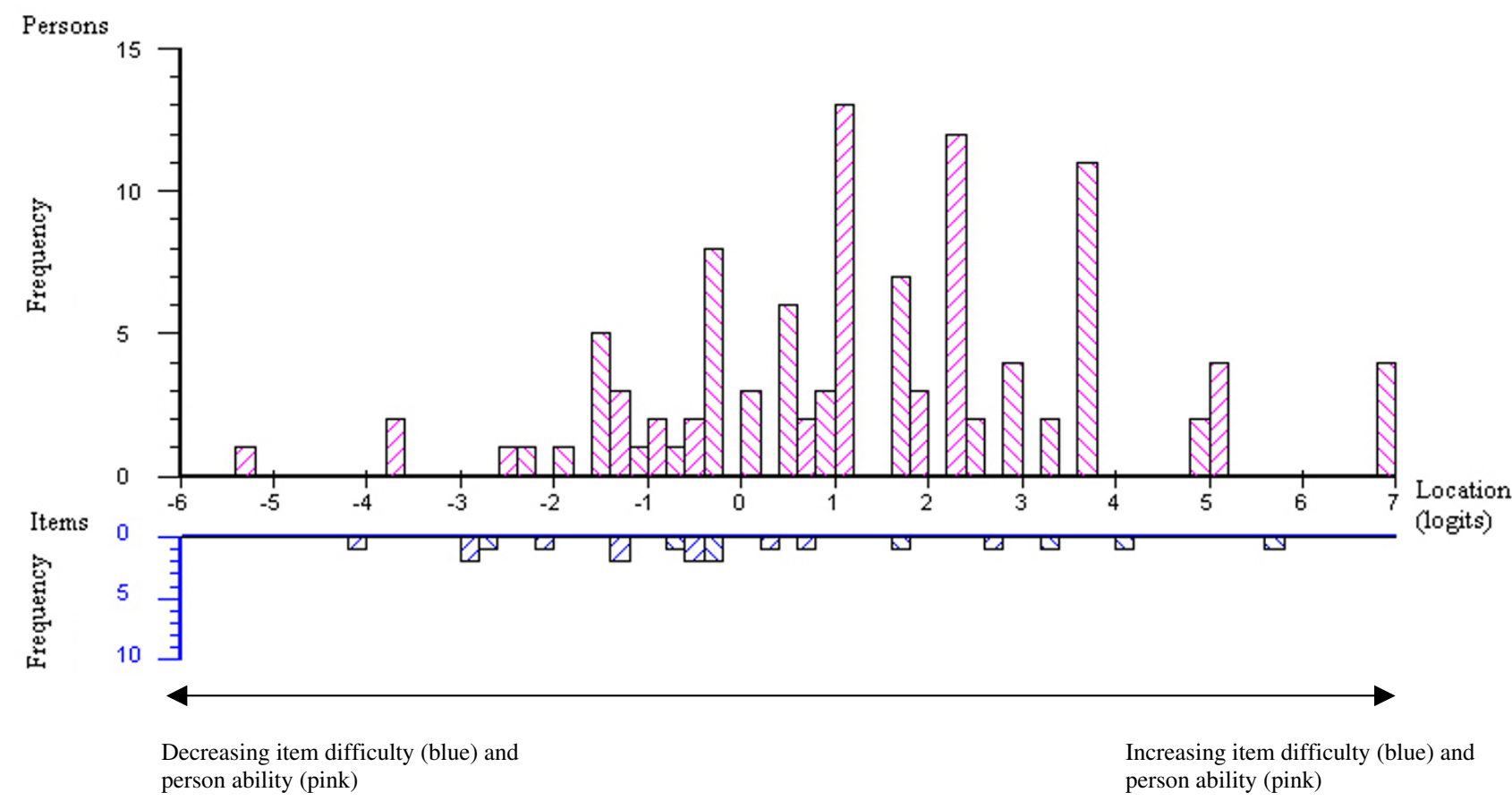

Figure 4

Person-item threshold graph for admission mobility assessments for the I 5 item DEMMI in the validation sample. 


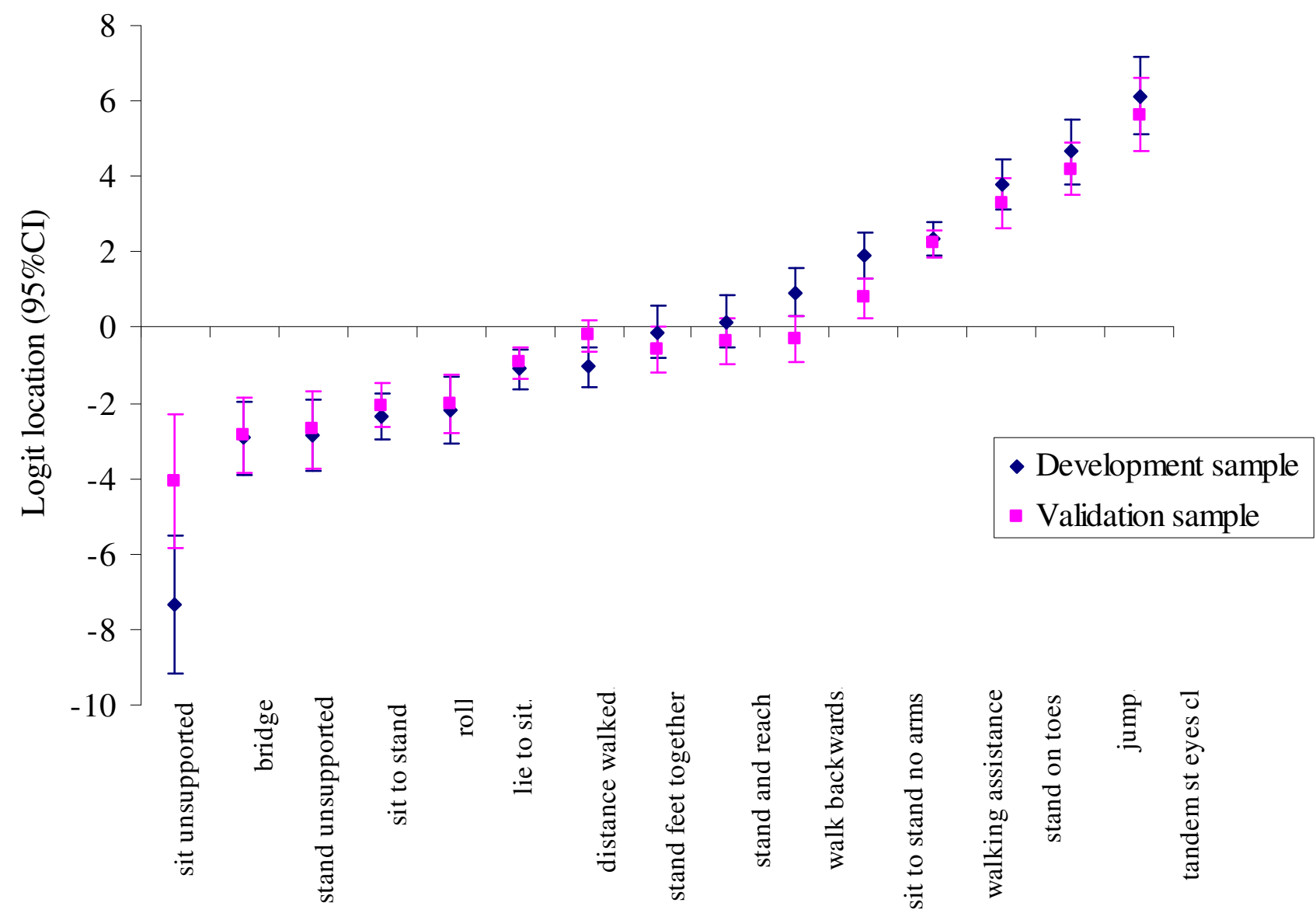

Figure 5

Item logit location for baseline data for the scale development and validation studies.

improvement and deterioration in mobility across the spectrum of mobility levels that exist in an older acute medical patient population.

The DEMMI contains items that are considered to be important hallmarks of independent mobility and have face validity for measuring the domain of mobility as defined by the World Health Organisation [14]. Therefore this new mobility instrument facilitates the comprehensive assessment of mobility for older medical patients and assessment findings can be used to assist in goal setting for therapeutic intervention. For example, an older medical patient who has a logit location of -2.4 (or interval measure score of 38 at hospital admission) would be expected to be able to perform bed based mobility tasks, require minimal assistance or supervision for transfers in and out of the chair, have adequate balance to sit and stand unsupported and walk short distances with assistance or supervision. The mobility hierarchy indicates that for this patient to progress along the mobility continuum, goals for therapeutic intervention should include achieving independence in bed and chair transfers, then increasing walking distances and improving standing balance. Any item that the patient cannot do that they would be expected to be able to complete based on their total score can also be easily identified using this method (i.e. items lower in the mobility hierarchy than other items that were successfully completed).

The DEMMI has minimal equipment requirements and the scale protocol and scoring system fit onto one page (back and front). Only a bed or plinth, arm chair (seat height $45 \mathrm{~cm}$ ) and pen are required to conduct the test. These pieces of equipment are usually readily available in hospital wards or emergency departments. The DEMMI can be quickly and easily applied in an acute hospital where the time and space available for testing would be similar, if not more constrained, than other clinical settings. Since the DEMMI is appropriate and without practical limitations for the broad spectrum of conditions seen in older acute medical patients, it is likely to also be safe for administration in most clinical populations. 
The DEMMI item hierarchy was consistent across independent samples despite being administered by differing clinicians and testing a smaller number of items in the validation study. In support of earlier studies [5,34], this provides strong evidence that for heterogeneous older patient populations, physical recovery from illness follows a common path. Since older patients are expected to progress across the DEMMI mobility continuum in a predictable manner, the DEMMI hierarchy provides clinicians with a systematic method for identifying capabilities and limitations. The DEMMI facilitates comprehensive assessment of mobility and assessment findings can be used to define specific targets for therapeutic intervention.

Fit of the data to the Rasch model validates the summation of mobility item scores to produce a total mobility score and indicates that the DEMMI provides interval level data. A simple conversion table allows ordinal mobility scores (out of 19) to be converted to interval mobility scores (out of 100). For statistical purposes, a group mean increase of 20 (converted) points, for example, represents the same amount of improvement in mobility across individuals regardless of whether the patient is bed bound or independently mobile at initial assessment. Interval data allows researchers to interpret parametric statistical tests of DEMMI data.

In the validation study, two items were tested but removed from analysis. The jog item was removed to maximise the potential for the DEMMI to be used by clinicians with varying clinical experience and from different healthcare disciplines. Since this item was tested last, patient performance on this item did not influence performance on other items. The standing on one leg eyes closed item was the most difficult item in the instrument development sample. Since no participants were able to successfully complete the standing on one leg eyes closed item in the validation study, this extreme item could not be included in Rasch analysis. However, given that the properties of the 15 DEMMI items were consistent across independent samples despite the differing number of items tested, removal of this item (attempted by only 30\% of patients) is unlikely to have influenced the estimated clinimetric properties of the final instrument.

The consistency of the DEMMI across independent samples provides confidence in the interpretation and clinical application of DEMMI scores. The $\mathrm{MDC}_{90}$ indicates that a minimum change score of 9 Rasch converted points on the DEMMI is required for $90 \%$ confidence that a true change in patient mobility has occurred and the MCID indicates a minimum change of 10 points is required to represent a clinically important change in patient mobility. These data were derived from inter-rater error esti- mates. Since inter-rater reliability estimates are typically larger than intra-rater, our calculations provide clinicians with conservative estimates of measurement error.

Although the DEMMI was developed for acutely hospitalised older patients, the potential applications of this instrument are broad. Due to its inclusive scale width, the DEMMI has the potential to be used in many clinical settings and subsequently enhance the continuity of care of older adults across providers, clinical settings and in the community. Further research is underway to validate the DEMMI across clinical settings and in the community and to translate the DEMMI into other languages.

It is possible that sampling bias may exist in the data reported in this research. Firstly, the requirement to obtain written and informed consent may have resulted in the inclusion of a healthier and less cognitively impaired cohort of patients compared to a typical older acute medical population. Secondly, data collection for both the development and validation studies was conducted at the same hospital site. However, since Rasch analysis assesses the consistency of item response patterns relative to the total score, sampling bias will not have influenced the fit of the data to the Rasch model in this research.

\section{Conclusion}

The DEMMI has been rigorously developed and validated. More than 500 DEMMI assessments have been conducted and the Rasch, reliability, validity and MCID properties of the DEMMI were consistent across independent samples of older acute medical patients. Maximising the independence of older people is fundamental to prolonging health and quality of life and reducing dependence on limited healthcare resources.

\section{Competing interests}

The authors declare that they have no competing interests.

\section{Authors' contributions}

$\mathrm{Nd}$ conceived and designed the study, acquired the data, analysed and interpreted the data, wrote the manuscript and has given final approval of the version to be published. MD contributed to the analysis and interpretation of the data, has been involved in the drafting of the manuscript and given approval for the version to be published. JK contributed to the conception and design of the study, the analysis and interpretation of data, drafting of the manuscript and has given final approval of the version to be published.

This research was presented by Dr Natalie de Morton at the World Physical Therapy Congress, Vancouver, Canada, June 2007, the Australian Physiotherapy Association Confer- 
ence, Cairns, Australia, October 2007 and the Australian Association of Gerontology Conference (NSW region), Woolongong, Australia, April 2008.

\section{Additional material}

\section{Additional file 1}

The DEMMI.

Click here for file

[http://www.biomedcentral.com/content/supplementary/14777525-6-63-S1.pdf]

\section{Acknowledgements}

The authors would like to acknowledge the support of The Northern Clinical Research Center, Northern Health (in particular, Dr David Berlowitz, Ms Marnie Graco, Ms Anna Barker, Mr Shane Grant, Ms Victoria Lawlor and Ms Dorothy Lewis) and the physiotherapy department at The Northern Hospital, Northern Health.

Funding sources for this research were the HCF Health and Medical Research Foundation (external grant) and the National Health and Medical Research Council of Australia (Dora Lush Postgraduate Scholarship, Grant no. 280632)

\section{References}

I. Mazzeo R, Cavanagh P, Evans W, Fiatarone M, Hagberg J, McAuley E, Startzell ]: ACSM Position Stand: Exercise and Physical Activity for Older Adults. Medicine \& Science in Sports \& Exercise 1998, 30:992-1008.

2. Morris ME, Schoo Ae: Optimizing Exercise and Physical Activity in Older People London, UK: Butterworth Heinemann; 2004.

3. de Morton N, Berlowitz D, Keating J: A systematic review of mobility instruments and their measurement properties for older acute medical patients. BMC Health and Quality of Life Outcomes 2008, 6:44.

4. Smith R: Validation and reliability of the Elderly Mobility Scale. Physiotherapy 1994, 80:744-747.

5. MacKnight C, Rockwood K: Rasch analysis of the hierarchical assessment of balance and mobility (HABAM). Journal of Clinical Epidemiology 2000, 53: I 242-1247.

6. Winograd CH, Lemsky CM, Nevitt MC, Nordstrom TM, Stewart AL, Miller CJ, Bloch DA: Development of a physical performance and mobility examination. Journal of the American Geriatrics Society 1994, 42:743-749.

7. Podsiadlo D, Richardson S: The Timed "Up \& Go": a test of basic functional mobility for the frail elderly persons. Journal of the American Geriatrics Society 1991, 39:|42-|48.

8. Mahoney FI, Barthel DW: Functional Evaluation: The Barthel Index. Maryland State Medical Journal 1965, I 4:61-65.

9. de Morton NA, Keating JL, Berlowitz DJ, Jackson B, Lim WK: Additional exercise does not change hospital or patient outcomes in older medical patients: a controlled clinical trial. Australian Journal of Physiotherapy 2007, 53:105-III.

10. de Morton N, Jones C, Keating J, Berlowitz D, MacGregor L, Lim W, Jackson B, Brand C: The effect of exercise on outcomes for hospitalised older acute medical patients: An individual patient data meta-analysis. Age Ageing 2007, 36:219-222.

I I. de Morton NA, Keating JL, Jeffs K: Exercise for acutely hospitalised older medical patients. Cochrane Database Syst Rev 2007, 24(I):CD005955.

12. de Morton NA, Keating JL, Jeffs K: The effect of exercise on outcomes for older acute medical inpatients compared to control or alternative treatments: a systematic review of randomised controlled trials. Clinical Rehabilitation 2007, 2 I:3-I6.

13. de Morton N, Keating J, Davidson M: Rasch analysis of the Barthel Index in the assessment of hospitalised older patients follow- ing admission for an acute medical condition. Archives of Physical Medicine \& Rehabilitation 2008, 89:64I-647.

14. World Health Organisation: International Classification of Functioning, Disability and Health Geneva, Switzerland; 2001.

15. Rikli RE, Jones CJ: Assessing Physical Performance in independent older adults: Issues and guidelines. Journal of Aging and Physical Activity 1997, 5:244-26I.

16. Chodzko-Zajko WJ: Assessing physical performance in older adult populations. Journal of Aging and Physical Activity 1994, 2:103-104.

17. Guralnik J, Simonsick E, Ferrucci L, Glynn R, Berkman L, Blazer D, Scherr $\mathrm{P}$, Wallace $\mathrm{R}$ : A short physical performance battery assessing lower extremity function: association with selfreported disability and prediction of mortality and nursing home admission. J Gerontol I994, 49(2):M85-M94.

18. Folstein MF, Folstein SE, McHugh PR: "Mini-Mental State." A practical method for grading the cognitive state of patients for the clinician. Journal of Psychiatric Reserve 1975, 12:189-198.

19. Charlson ME, Pompei P, Ales KL, MacKenzie CR: A new method of classifying prognostic comorbidity in longitudinal studies: development and validation. Journal of Chronic Disease 1987, 40:373-383.

20. Knaus W, Draper E, Wagner DP, Zimmerman JE: APACHE I I: A severity of disease classification system. Critical Care Medicine 1985, 13:818-829.

21. Shah S, Vanclay F, Cooper B: Improving the sensitivity of the Barthel Index for stroke rehabilitation. Journal of Clinical Epidemiology 1989, 42:703-709.

22. SPSS for Windows [computer program]. Chicago: SPSS Inc; 2003. Rel 12.0 .0

23. Andrich D, Sheridan BS, Luo G: RUMM2020 [computer program]. Western Australia: RUMM Laboratory.

24. Smith E: Detecting and evaluating the impact of multidimensionality using item fit statistics and principal components analysis of residuals. Journal of Applied Measurement 2002, 3:205-23I.

25. Smith R: Fit analysis in latent trait measurement models. Appl Meas 2000, I(2): 199-218.

26. Linacre JM: Sample size and item calibration stability. Rasch Measurement Transactions 1994, 7:328.

27. Wright BD: Rack and stack: time I vs time 2. Rasch Measurement Transactions 2003, I 7:905-906.

28. Chang W, Chan C: Rasch analysis for outcome measures: some methodological considerations. Archives of Physical Medicine and Rehabilitation 1995, 76:934-939.

29. Walter SD, Eliasziw M, Donner A: Sample size and optimal designs for reliability studies. Statistics in Medicine 1998, 17:101-110.

30. Stratford PW, Binkley JM, Riddle DL: Health status measures: strategies and analytic methods for assessing change scores. Physical Therapy 1996, 76: I 109-I I 23.

31. Norman GR, Sloan JA, Wyrwich KW: Interpretation of changes on health related quality of life. The remarkable universality of half a standard deviation. Medical Care 2003, 41:582-592.

32. Tryon W: Evaluating statistical difference, equivalence, and indeterminacy using inferential confidence intervals: an integrated alternative method of conducting null hypothesis statistical tests. Psychological Methods 2001, 6:371-386.

33. Tennant A, Pallant J: Unidimensionality matters! (A tale of two Smiths?). Rasch Measurement Transactions 2006, 20: I048-I05I.

34. Katz S, Ford A, Moskowitz R, Jackson B, Jaffe M: Studies of illness in the aged. Journal of the Amercian Medical Association 1963 1 85:94-99. 